\title{
ZUR BALTISTIK IN SCHWEDEN UM DIE MITTE DER SIEBZIGER JAHRE DES VERGANGENEN JAHRHUNDERTS
}

\author{
Rainer ECKERT \\ Berlin
}

\section{Vorbemerkung}

Ich widme diesen Vortrag meinem lieben Kollegen Prof. Dr. Trevor Fennell zum 80. Geburtstag und sende ihm herzliche Grüße und alle guten Wünsche. Wir haben uns auf vielen internationalen Konferenzen zur Baltistik in Schweden, in Deutschland, in den baltischen Staaten und ziemlich regelmäßig auf den internationalen Kongressen der AABS von 1992 bis 2006 in Nordamerika getroffen und pflegten einen herzlichen und regen Austausch. Besonders gern erinnere ich mich an unsere gemeinsame Busfahrt von Indianapolis nach Bloomington am 18. Juni 1989 zur 16. AABS-Konferenz „Baltische Probleme in internationalen Perspektiven“ vom 19. bis 21. 06. 1989 an der Indiana University Bloomington. ${ }^{1}$

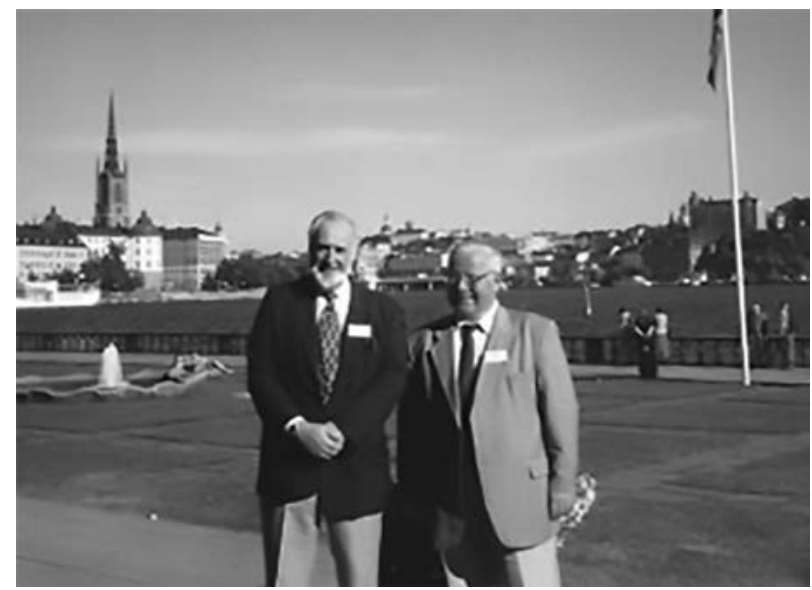

$A b b$. 1. Prof. Dr. Trevor Fennell (links) mit dem Autor in Stockholm vor dem Rathaus am Mälarsee, 1989

1 Rainer Eckert. Die 20. Konferenz der AABS in Washington 2006 (Bericht mit anschließender Zusammenfassung meiner Kongressreisen nach Amerika), in: Baltu filolog̣ija XV (1/2) Rīga 2006, 159. 
Lieber Trevor, ich möchte Sie meines tiefsten Dankes für die vielen wertvollen Buchgeschenke zur Geschichte des Lettischen und vor allem zur historischen Lexikographie dieser Sprache versichern und gleichzeitig bekunden, dass ich die faszinierenden und gehaltvollen Gespräche mit Ihnen in freudiger Erinnerung bewahre.

Ausgangspunkt für den vorliegenden Beitrag war eine freundliche Einladung der Leiterin des Baltistischen Bereiches des Instituts für slawische und baltische Sprachen (Institutionen för slaviska och baltiska språk) der Universität Stockholm Frau Prof. Dr. Velta Rūkse-Dravina zu Vorlesungen über slawische Phraseologie und zur 4. Konferenz über Baltistik in Schweden mit einem Studienaufenthalt in Stockholm vom 04. bis 11. Oktober 1976. Meine Eindrücke über die schwedische Baltistik unter Auswertung des Artikels von Frau Prof. V. Rūķe-Draviņa „Lettisk och litauisk språkforskning“ (,Lettische und litauische Sprachforschung ") regten mich an, einen Vortrag im Berliner Baltistenkreis $^{2}$ zu halten, der leider damals nicht veröffentlicht wurde, da wir kein Publikationsorgan für unsere wissenschaftliche Gesellschaft besaßen.

\section{Zu einigen markanten Zügen der schwedischen Baltistik}

Die schwedische Baltistik nimmt eine beachtete Stellung innerhalb der Baltistik der skandinavischen Länder ein und lässt eine wachsende Bedeutung für die internationale Entwicklung dieses sprachwissenschaftlichen Zweiges erkennen. So finden seit Beginn der siebziger Jahre in einem Zweijahresrhythmus Symposien zur Baltistik an der Universität Stockholm statt. Die erste Konferenz dieser Art fand im Jahre 1971 statt als Symposium om balterna i Sverige. Föredrag hållna vid symposiet den 4-5 juni 1971 i Stockholm på Hässelby slott. Es folgte das zweite Symposium als Second Conference on Baltic Studies in Scandinavia 1973, das dritte Symposium als The Third Conference on Baltic Studies in Stockholm 1975 und das vierte Symposium als The Fourth Conference of Baltic Studies in Scandinavia 1977.

Seit dem Jahre 1970 hat an der Stockholmer Universität ein Baltisches Institut (Baltiska Institutet) seinen Sitz. In den erwähnten Symposien spielten vor allem die gegen Ende des Zweiten Weltkrieges vor der Roten Armee geflüchteten und emigrierten Wissenschaftlerinnen und Wissenschaftler aus Lettland und Litauen eine bedeutende Rolle, deren Tätigkeit eine längere Zeit in der Sowjetunion und den ihr ideologisch angeschlossenen Ländern

2 Es handelt sich um den Vortrag „Die Baltistik in Schweden" auf der 36. Baltistenkonferenz am 18. März 1977. Rainer Eckert. Der Baltistenkreis zu Berlin e.V. und seine Vorgängereinrichtung, Peter Lang Verlag, Frankfurt am Main 2015, 128. 
ignoriert wurde. Doch in den sechziger und darauf folgenden Jahren kam es zu gewissen Entspannungen und sich mehrenden Kontaktaufnahmen in der Wissenschaft, dem natürlich die neutrale Politik Schwedens entgegen kam.

Dies zeigte sich vor allem in der Teilnahme einer größeren Gruppe von Wissenschaftlerinnen und Wissenschaftlern an der überragenden Festschrift für Prof. Dr. Chr. S. Stang ${ }^{3}$. Von insgesamt 75 Autoren, die sich an diesem Werk beteiligten, stammt mehr als ein Drittel (genau 27) aus der Sowjetunion und den mit ihr liierten Ländern. Besonders aufschlussreich ist die Beteiligung von 10 Autoren aus Litauen und Lettland.

Wie in vielen Ländern ist die Baltistik eng mit der Slawistik verbunden. Das galt in den vergangenen Dezennien für die Universitäten in Lund, Uppsala und Stockholm, wobei besonders in den letzten Jahren ein Trend zu einer gewissen Eigenentwicklung vor allem in Stockholm auszumachen ist.

Außerdem ist zu beachten, dass das Finnische eine besondere Rolle in den schwedischen Hochschulen spielte, vor allem an der Universität in Umeå. Auch das Westfinnische (hier natürlich Estnisch) und das Saamische ergänzen das Interesse am Finno-ugrischen in der schwedischen Wissenschaftslandschaft.

Eine besondere Situation für die Baltistik in Schweden war in der Nachkriegszeit durch den Zustrom einer bedeutenden Anzahl baltischer Emigranten zu verzeichnen. So kamen aus Estland und Lettland etwa je 4.500 Emigranten, aus Litauen etwa 400. Dieser Umstand hatte einen gewissen Einfluss auf die Entwicklung und Etablierung von Lehr- und Forschungseinrichtungen für baltische Philologie. Eine Reihe von Wissenschaftlern und Lehrkräften aus Lettland und Litauen nahm ihre Tätigkeit auf. Aber auch viele Hörerinnen und Hörer kamen aus Emigrantenkreisen, zunehmend auch aus anderen westlichen Ländern, in die Balten emigriert waren.

Kennzeichnend ist weiterhin für die Baltistik in Schweden eine deutliche Orientierung auf das Studium der baltischen Gegenwartssprachen Lettisch und Litauisch, ihre Literaturen sowie ihre Geschichte und Landeskunde. Zumindest an der Universität Stockholm wird die Baltistik als eigenständiger Lehr- und Forschungsgegenstand betrieben und nicht wie andernorts in früheren Zeiten als Anhängsel der Indogermanistik oder Slawistik mit einer deutlichen Reduzierung auf das Litauische. Gleichzeitig ist zu betonen, dass in Schweden der historischen Komponente des Baltischen (Sprach-, Literatur- und Landesgeschichte, Folklore) die gebührende Aufmerksamkeit geschenkt wird. Das Lettische wurde in diesem Zusammenhang gleichrangig zum Litauischen entwickelt und behandelt.

3 Donum Baltikum. To professor Christian S. Stang on the occasion of his seventieth birthday, 15 March 1970. Edited by Velta Rūķe-Draviņa, Stockholm1970, 598 S. 


\section{Zu einigen Traditionslinien}

Die Baltistik in Schweden besitzt, wie in einer Reihe anderer Länder, in der Vergangenheit eine nicht unbedeutende Tradition, aus der hier aus Raumgründen nur einige wenige Momente dargestellt werden können. Kennzeichnend war auch hier in der Regel ein engerer Bezug zur Indoeuropäistik und/oder zur Slawistik.

Prof. Tore Torbjörnsson veröffentlichte bereits 1901 in Uppsala seine zweibändige, grundlegende Studie „Die gemeinslawische Liquidametathese“, die auch ein reiches baltisches Material enthält.

An der Universität Uppsala war Prof. Richard Ekblom erfolgreich auf slawistischem und baltistischem Gebiet tätig. Er arbeitete mit Prof. Jānis Endzelīns von der Universität Riga und mit dem Phonetischen Institut der Universität Stockholm zusammen bei der experimental-phonetischen Untersuchung der lettischen Intonation. Im Jahre 1933 erschien dazu sein Buch „Die lettischen Akzentarten“. ${ }^{4}$

Prof. Knut-Olaf Falk hatte in den 20er und 30er Jahren das Lektorat für Schwedisch an der Universität Kaunas inne. Er trat später mit einer Reihe von baltistischen Arbeiten, darunter Beiträgen zur Slawisierung litauischer Ortsnamen und Antroponyma, auf und wirkte lange Jahre als Baltist an der Universität Lund. ${ }^{5}$ Er und Prof. Dr. J. Safarewicz hatten Anteil an der Gründung der Kommission zum Studium der balto-slawischen Beziehungen beim Internationalen Slawistenkomitee 1963.

\section{Zur weiteren Entwicklung der schwedischen Baltistik in der Nachkriegszeit bis in die 70er Jahre}

Aus dem Dargestellten wird deutlich, dass es in der Nachkriegszeit in der schwedischen Baltistik wichtige Impulse in vor allem drei Richtungen gab:

1) in einer auf das praktische Sprachstudium ausgerichteten Lehre, wobei, wie bereits erwähnt, das Lettische gleichberechtigt neben dem Litauischen behandelt wurde und die spezielle Beziehung zum Livischen die Beachtung des Finnougrischen verstärkte;

4 Bereits 1927 war in Uppsala-Leipzig seine Arbeit „Zur Physiologie der Akzentuation langer Silben im Slawo-Baltischen“ erschienen.

5 Man vgl. „Ze studiów nad slawizacją litewskich nazw miejscowych i osobowych: O wtórnej funkcji sufiksu -ec; o nazwach miejscowych na -ance“. Scando-Slavica, IV (1963), 87-103; „O metodach slawizacji litewskich nazw osobowych i miejscowych, o genezie i rozpowszechnieniu nazw na -ance“. Språkliga bidrag, vol. 9, Lund 1966, 1-16. 
2) eine Ausweitung auf die verschiedensten Teilgebiete der Sprachwissenschaft (über die Lautlehre, Akzentologie, Grammatik und Lexik hinaus), auf Bereiche der Dialektologie, Onomastik, Geschichte der Schriftsprachen, Phraseologie, Sprache der Folklore, Kindersprache etc.;

3) eine vertiefte Erforschung der baltisch-slawischen Sprachbeziehungen.

Hier sind vor allem die zahlreichen Arbeiten von Prof. P. Arumaa, K. Draviņš und Prof. V. Rūķe-Draviņa zu nennen.

Der aus Estland stammende Prof. Peeter Arumaa war an den Universitäten Lund und Stockholm tätig und befasste sich u.a. mit der Erforschung der litauischen Dialekte ${ }^{6}$, der Geschichte der litauischen Personalpronomina ${ }^{7}$ und der Nominalstämme auf $-u$ im Baltischen und Slawischen ${ }^{8}$. Einen bedeutenden Platz in seiner dreibändigen „Urslavischen Grammatik“9 nimmt das Baltische ein. Er äußerte sich auch in mehreren Arbeiten speziell zum Balto-Slawischen. ${ }^{10}$

Kārlis Draviņš, der an der Universität Lund angestellt war, gab ein wichtiges Sprachdenkmal des Altlettischen ${ }^{11}$ heraus und veröffentlichte zusammen mit seiner Frau Velta Rūkse eine Serie von Beschreibungen über die Mundart seiner Heimat Stenden ${ }^{12}$.

Prof. Dr. V. Rūķe-Draviņa veröffentlichte 1959 ihre Habilitationsschrift über die Diminutive im Lettischen ${ }^{13}$, ein Standardwerk zu dieser für das Baltische (und Slawische) so charakteristischen Kategorie. Im Jahre 1949 hatte sie einen Beitrag, den sie „Die Muttersprache in der Emigration“

${ }_{6}$ Peeter Arumaa. Litauische mundartliche Texte aus der Wilnaer Gegend. Dorpat 1930.

7 Peeter Arumaa. Untersuchungen zur Geschichte der litauischen Personalpronomina, Tartu 1933.

${ }^{8}$ Peeter Arumaa. Sur l'histoire des adjectifs en - $u$ en balto-slave. Årsbok 1948-1949, Lund 1951.

9 Peeter Arumaa. Urslavische Grammatik. Bde 1-3. Heidelberg 1964-1982.

${ }^{10}$ Ich verweise nur auf zwei Arbeiten: Die Verwandtschaftsverhältnisse zwischen Baltisch und Slavisch. Zeitschrift für slavische Philologie, Bd. 24 (1956), 9-28 und De l'unité balto-slave. Scando-Slavica, t. IX (1963), 70-86.

${ }^{11}$ Evangelien und Episteln, ins Lettische übers. von Georg Elger, Bd. 1. Texte. Herausgegeben von K. Draviņš, Lund 1961.

${ }^{12}$ Man vgl. die Hefte der Slaviska och baltiska studier, Slaviska institutionen vid Lunds Universitet:

(1) K. Draviņš \& V. Rūķe. Laute und Nominalformen der Mundart von Stenden. 1. Einleitung, Akzent und Intonation, Lautlehre, 1955; (2) Dieselben. Laute und Nominalformen der Mundart von Stenden. 2. Wortbildung, Deklination und Komparation, 1956; (3) Dieselben. Verbalformen und undeklinierbare Redeteile der Mundart von Stenden. Verben, Adverbien, Präpositionen und Präfixe, Partikeln, Konjunktionen, 1958 und (4) Dieselben. Interjektionen und Onomatopöie in der Mundart von Stenden, 1962.

13 Velta Rūkse-Draviņa. Diminutive im Lettischen. Lund 1959, 408 S. 
nannte, veröffentlicht, der treffend die Problematik einer ganzen Reihe von Beiträgen aus ihrer Feder in dieser Zeit umriss. In den Jahren von 1945 bis 1975 brachte sie, vorwiegend in Schweden, aber auch in einer Reihe anderer Länder 127 Arbeiten heraus, die ein breites Feld der Baltistik betreffen. Im Mittelpunkt steht natürlich das Lettische, das aus den verschiedensten Blickwinkeln einer Betrachtung unterzogen wird, wobei die einzelnen Beiträge weit über die Fragen der Struktur der baltischen Sprachen hinausreichen. Es werden Probleme der Onomastik, des Spracherwerbs und der Kindersprache, der Übersetzungswissenschaft und vieles andere behandelt. Ein hoher Grad von Universalität ihrer gesamten Sprachbetrachtung deutet sich an. Im Jahre 1974 brachte Prof. Rūkse-Draviņa das erste phraseologische Wörterbuch des Lettischen in Schweden heraus. ${ }^{14}$ Sie zählt zu den Begründern dieser jungen sprachwissenschaftlichen Disziplin.

In enger Beziehung zu den erfolgreichen Forschungen steht bei Prof. Rūķe-Draviņa ihre beispielhafte, viele Teildisziplinen umfassende Lehre an den Hochschulen. Sie hielt von 1948 bis 1968 Lehrveranstaltungen zu den baltischen Sprachen an der Universität Lund und hatte von 1969 bis 1984 die Leitung der Baltistik an der Stockholmer Universität inne, die sie während ihrer Tätigkeit zu einer der führenden Ausbildungsstätten der Baltistik in Europa und in der Welt entwickelte. In dieser Zeit belegten bis zu 20 Studenten und Doktoranden aus vielen Ländern jährlich das Baltistikstudium in Stockholm. Als Schülerin von Prof. Dr. J. Endzelīns, Mitglied der Königlichen Schwedischen Humanistischen Akademie der Wissenschaften seit 1980 und Trägerin weiterer zahlreicher Ehrungen zählt sie zu den hervorragendsten Vertretern in der Geschichte der Baltistik.

\section{Zur Zusammenarbeit von Baltisten und Slawisten aus Schweden und der DDR}

Eine erste wissenschaftliche Zusammenarbeit auf dem Gebiete der Slawistik setzte relativ früh in der Nachkriegszeit ein. Hier wären vor allem die Kontakte von Prof. Dr. Rudolf Růžička und Prof. Dr. Heinz SchusterŠewc zu den Universitäten Stockholm und Göteborg zu erwähnen. Die schwedischen Professoren Anders Sjöberg (Universität Stockholm), Gunnar Jacobsson (Universität Göteborg) und Thorsten Andersson (Universität Uppsala) besuchten die Universität Leipzig.

14 Velta Rūḳe-Draviņa. Vārds īstā vietā. Frazeolog̣ismu krājums (Das richtige Wort am richtigen Fleck. Eine Sammlung von Phraseologismen), Stockholm 1974, 338 S. 
Seit dem V. Internationalen Slawistenkongress in Warschau 1973 konnte ich persönliche Kontakte mit Frau Prof. Dr. V. Rūkse-Draviņa aufnehmen. Im Jahre 1974 trat sie mit Gastvorlesungen an der Leipziger Universität auf. So hielt sie Vorlesungen zu den Zahlwörtern im Baltischen und zur konfrontativen Phraseologie im Lettischen, Litauischen und Deutschen sowie einem Vortrag über slawische Onomastik.

Von Frau Prof. Dr. Rūķe-Draviņa erhielt ich ab 1976 jeweils die Einladungen zu den Internationalen Baltistischen Konferenzen an der Universität Stockholm, für die ich ihr sehr dankbar war. 1989 führte ich als leitender Moderator das Promotionsverfahren von Frau Konstance Klava an der Universität Stockholm durch. Im gleichen Jahr hatte ich von der Universität einen dreiwöchigen Studienaufenthalt in Stockholm zu Forschungsarbeiten erhalten.

Auf der 79. Konferenz des Berliner Baltistenkreises hielt Frau Prof. Dr. Baiba Kangere einen Vortrag über die lettische und litauische Literatur im Exil der Jahre 1950 bis 1990 und Dr. Kārlis Kangeris (beide von der Universität Stockholm) referierte über „die Kulturpolitik der deutschen Okkupationsmacht im Generalkommissariat Lettland 1941-1945“. 1989 besuchte Frau Dr. Aija Priedīte Berlin. In den letzten Jahrzehnten gab es einen regen wissenschaftlichen Austausch und enge Kontakte zu Herrn Prof. Dr. Pēteris Vanags, dem ich sehr viel in meinem baltistischen Werdegang verdanke.

Im letzten Abschnitt bin ich weit über den zeitlichen Rahmen hinausgegangen, um zu zeigen, dass die in den siebziger Jahren geknüpften Kontakte zwischen den ostdeutschen und schwedischen Wissenschaftlern besonders nach der Wiedervereinigung Deutschlands 1989 weiter ausgebaut wurden und eine kontinuierliche Fortsetzung fanden. Mich persönlich erfüllt der vorliegende Bericht mit großer Genugtuung und Freude über die Gestaltung fruchtbarer Beziehungen zwischen der deutschen Baltistik und der elitären skandinavischen Baltistik (konkret der schwedischen Baltistik, die hier Gegenstand war mit dem noch zu ergänzenden Hinweis auf besonders tragende Kontakte zu solchen großen norwegischen Baltisten wie Prof. Dr. Christian S. Stang, Prof. Dr. Terje Matthiassen und Prof. Dr. Helge Rinholm).

Rainer Eckert

Emeritus

Murtzaner Ring 16

12681 Berlin, Deutschland

rainer_eckert@gmx.net 
KOPSAVILKUMS

\section{Par baltistikas pētījumiem Zviedrijā pagājušā gadsimta 70. gadu vidū}

\section{Rainer ECKERT}

Rakstā sniegts īss ieskats baltu valodu pētniecībā Zviedrijā 20. gadsimtā. Tuvāk apskatītas valodnieku aktivitātes septiņdesmitajos un astoņdesmitajos gados. Rakstā minētas nozīmīgākās publicētās grāmatas, kā arī Zviedrijā rīkotās zinātniskās konferences. Atsevišḳi aplūkota arī bijušās VDR un Zviedrijas baltistu un slāvistu sadarbība pagājušā gadsimta septiņdesmitajos un astonidesmitajos gados.

\section{SUMMARY}

\section{On Baltic Studies in Sweden around the Mid-Seventies of the Last Century}

\section{Rainer ECKERT}

The article provides a brief overview of research on the Baltic languages in Sweden in the 20th century. The activities of linguists in the 1970s and 1980s are examined in detail. The article mentions the most important books published, as well as scientific conferences on Baltic studies held in Sweden. The co-operation between Slavists and Balticists of the former GDR and Sweden in the 1970s and 1980s is also briefly discussed. 